\title{
Polarimetry as a window into supernova explosions and progenitors
}

\author{
Jennifer L. Hoffman \\ Department of Physics \& Astronomy, University of Denver \\ 2112 E. Wesley Ave., Denver, CO 80210, USA \\ email: jennifer.hoffman@du.edu
}

\begin{abstract}
Supernovae of all types exhibit time-dependent spectropolarimetric signatures produced primarily by electron scattering. These reveal the presence of aspherical and variable phenomena such as complex velocity structures, changing illumination, and asymmetric or clumpy morphologies within the ejecta or surrounding circumstellar material. In addition, the gradual thinning of the ejecta over time allows us to probe different scattering regions as the supernova evolves. Interpreting the time variations of these spectropolarimetric signatures yields unprecedentedly detailed information about supernova explosion mechanisms, the physical processes that shape the density and velocity distributions of the ejecta and circumstellar material, and the properties of the progenitor star.

I present an overview of supernova spectropolarimetry, highlighting recent observational and computational results. This versatile technique helps us to constrain explosion mechanisms, connect $\mathrm{SNe}$ with their massive progenitors (as well as other high-energy transient phenomena such as GRBs), and investigate the process of stellar evolution in other galaxies.
\end{abstract}

Keywords. polarization, instrumentation: polarimeters, techniques: polarimetric, catalogs, stars: mass loss, stars: winds, outflows, supernovae: general

\section{Introduction}

Supernovae (SNe) are broadly classified into two main types. Type Ia ("thermonuclear") SNe arise from white dwarf explosions, though the evolutionary scenarios giving rise to this occurrence are under debate (see, e.g., Tsebrenko \& Soker 2015). Supernovae of Types Ib/c and II ("core-collapse" SNe) arise from the collapse of massive stars after nuclear fusion ceases in their cores; in these cases, as well, the links between progenitor characteristics and SN subtypes are not well understood (Leonard 2011; van Dyk 2011). The wide variety of subcategories underlying these two types (see, e.g., Filippenko 1997 and Turatto et al. 2007) are distinguished by the details of their spectra and the behavior of their light curves over time.

Despite the differences among denizens of the supernova classification "zoo," SNe of all types have been shown to possess significant linear polarization signatures produced by electron scattering in their hot ejecta and surroundings (Wang \& Wheeler 2008). Different subtypes display differing polarimetric behavior, which changes over time as the shapes and configurations of the SN and its circumstellar material (CSM) evolve. Detailed investigation of these signatures can yield insights into the collapse and explosion mechanisms of supernovae (recently reviewed in Foglizzi et al. 2015 and Tsebrenko \& Soker 2015) and diagnose properties of the progenitor's pre-explosion winds, eruptions, and circumstellar material (e.g., Mauerhan et al. 2014). Because most supernovae are seen in faraway galaxies, spectropolarimetry of supernovae also allows us to test the efficacy of distance indicators that rely on assumptions of spherical symmetry (such as the expanding photosphere method, e.g., Leonard et al. 2002b), probe the characteristics 
of the dust in external host galaxies (Leonard et al. 2002a; Patat et al. 2015), and unveil the physical mechanisms of stellar evolution in the cosmological past.

Electron scattering, the primary polarigenic mechanism in $\mathrm{SNe}$, is a wavelength-independent process that produces linear polarization perpendicular to the source-scatterer plane. Because of this geometrical behavior, an unresolved spherical scattering region gives rise to zero net polarization. Measurements of a nonzero polarization signal that is constant with wavelength thus imply a globally aspherical or elongated scattering region. Such measurements have been used in SN studies to great effect, for example in attributing the dramatic increase in the polarization of the Type II-P SN 2004dj and related objects at the end of their plateau phase to an inner, aspherical core revealed by the decreasing optical depth of the outer ejected envelope (Leonard et al. 2006; Chornock et al. 2010; Dessart \& Hillier 2011; Leonard et al. 2012).

More complex wavelength-dependent effects, such as changes in polarization magnitude and/or position angle over an absorption or emission line, may be caused by clumps of material occulting the electron-scattering SN photosphere (Wang \& Wheeler 2008) or by resonant or fluorescent line scattering contributions to the polarization (Hoffman et al. 2008). In these cases, a careful analysis of the observed spectropolarimetric behavior can reveal geometrical details that are otherwise inaccessible. A few recent examples include the detection of a detached structure with a high Ca II optical depth moving rapidly toward the observer in the Type Ia SN 2001el (Kasen et al. 2003); signatures of a misalignment between the circumstellar material and the elongated ejecta in the Type IIn SN 1997eg (Hoffman et al. 2008); and jets and multi-component ejecta revealed by dramatic changes in polarization position angle in the same SN at different epochs (Kawabata et al. 2002; Maund et al. 2007).

Despite the power of these techniques, their application has been limited until recently by the small numbers of SNe studied with spectropolarimetry and the "snapshot" nature of the existing observations. Because SNe change rapidly during their early evolution, the true potential of spectropolarimetric SN studies can be unlocked only by long-term monitoring of these volatile objects (indeed, most of the advances described in this section relied on multiple epochs of observation). A dedicated, time-sensitive SN study promises to reveal significant new insights into the nature of SN explosions of all types.

\section{Supernova Spectropolarimetry with SNSPOL}

\subsection{The SNSPOL project}

The SNSPOL project is a recent and ongoing effort led by G. Grant Williams at MMTO and including collaborators at Steward Observatory, University of Denver, San Diego State University, UC Berkeley, and Université de Nice to compile an extensive database of the time-dependent spectropolarimetric behavior of SNe of all types. SNSPOL team members conduct monthly observations with the CCD Imaging/Spectropolarimeter (SPOL; Schmidt, Elston \& Lupie 1992; Mauerhan et al. 2014) at the 6.5-m Multiple Mirror Telescope (MMT) on Mt. Hopkins, the Kuiper 61-inch telescope on Mt. Lemmon, or the Bok 2.3-m telescope on Kitt Peak. This effort has yielded spectropolarimetric data on over 50 SNe since the project's inception; a detailed list of targets and observation dates is compiled on the SNSPOL website (http://grb.mmto.arizona.edu/ ggwilli/snspol/).

A major strength of the SNSPOL strategy is that the monthly observation schedule allows us to monitor the spectropolarimetric evolution of our targets over their optically visible lifetimes. We have more than one epoch of data for most of our targets, and 10-30 epochs for a few of particular interest (Table 1). This provides us with an unprecedentedly 
Table 1. SNe with spectropolarimetric data obtained by the SNSPOL project at 10 or more epochs, as of December 2014. Data from the SNSPOL website, http://grb.mmto. arizona.edu/ ggwilli/snspol/.

\begin{tabular}{|c|c|}
\hline | Туре & Epochs ${ }^{1}$ \\
\hline |2009ip ${ }^{2}$ | IIn & 12 \\
\hline |2010j1 $\quad$ | IIn & 30 \\
\hline | $2011 \mathrm{fe}^{4} \quad$ Ia & 16 \\
\hline | 2012A | II-P & 22 \\
\hline$\left|2012 \mathrm{au}^{5}\right| \quad \mathrm{Ib}$ & 19 \\
\hline | 2012cg | Ia & 11 \\
\hline | 2012ес | II-P & 20 \\
\hline | 2012fg | IIb & 12 \\
\hline | 2013ab | II-P & 12 \\
\hline | 2013bi | II-P & 11 \\
\hline | 2013ej ${ }^{6}$ II-P & 11 \\
\hline | 2012fh | Ic & 16 \\
\hline | 2013am | II & 12 \\
\hline | 2014L | Ic & 10 \\
\hline | 2014ab | IIn & 17 \\
\hline
\end{tabular}

Notes: ${ }^{1}$ Epochs refer to the number of different nights a target was observed. Some SNe were observed multiple times per night. ${ }^{2}$ Mauerhaen et al. (2014); ${ }^{3}$ Williams et al. (2014); ${ }^{4}$ Milne et al. (2014); ${ }^{5}$ Hoffman et al. (2014); ${ }^{6}$ Khandrika et al. (2014).

detailed view of the geometrical evolution of the ejecta and circumstellar material of a wide variety of SNe.

The SNSPOL collaboration also includes the MOunt LAguna SUpernova Survey (MOLASUS) group at San Diego State University, which performs optical and near-IR photometric monitoring of selected SNe using Mount Laguna Observatory's 1-meter telescope (Khandrika et al. 2014). We obtain supporting spectropolarimetric observations of Milky Way probe stars with the University of Wisconsin's HPOL spectropolarimeter at Ritter Observatory (Davidson et al. 2014) to constrain the contributions of Galactic interstellar polarization (ISP) to the observed SN polarization. The groups at the Université de Nice and University of Denver also contribute computational modeling of the observed SN data (e.g., Dessart \& Hillier 2011; Huk et al. 2013), an effort that will ramp up in the near future as the project shifts from data collection to analysis.

\subsection{SNSPOL results for SN 2009ip}

SNSPOL observations of the peculiar transient SN 2009ip shed new light on the question of whether its first 2012 brightness peak was an actual SN explosion (Mauerhan et al. 2013; Levesque et al. 2014) or simply another in a series of nonterminal LBV eruptions (Fraser et al. 2013; Margutti et al. 2014). The time-dependent spectropolarimetric data we obtained showed that the two 2012 flux peaks were characterized by two different and orthogonal polarization position angles (Figure 1; Mauerhan et al. 2014). This suggests that two distinct scattering regions produced the polarized signal at these two epochs.

After analyzing these results (Mauerhan et al. 2014), we concluded that the flux at the 2012a peak arose from the maximum brightness of a true core-collapse SN explosion and 

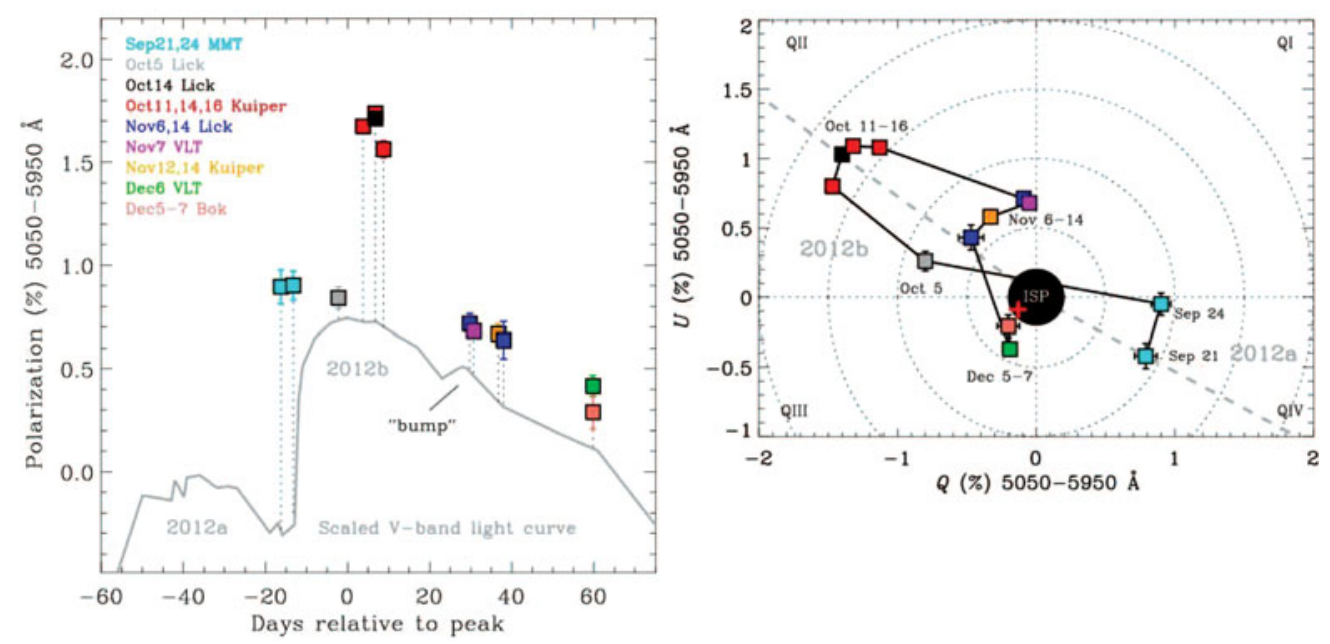

Figure 1. Left: Temporal evolution of the total $V$-band polarization for SN 2009ip during the $2012 \mathrm{a}$ and $2012 \mathrm{~b}$ phases. Filled squares represent polarization measurements; the gray line represents an arbitrarily scaled version of the $V$-band light curve. Right: Filled squares represent the same polarization measurements as at left, now plotted in the $Q-U$ plane. ISP is constrained to be less than $0.2 \%$, illustrated by the black dot near the origin. The dashed lines represent the position angles $\theta_{V}=166^{\circ}$ and $72^{\circ}$, as measured at the first epoch on Sep. 21 and peak polarization on Oct. 14, respectively. Our latest continuum measurement for Dec. 6 is marked with a cross near the origin, consistent with our ISP limit. The approximate point-reflection symmetry between 2012a and the peak of $2012 \mathrm{~b}$ suggests two separate and roughly orthogonal components of polarization on the sky. Note the temporary shift in $\theta$ associated with the bump in the light curve at $\sim 30 \mathrm{~d}$ past peak. Figure from Mauerhan et al. (2014), courtesy Oxford University Press and the Royal Astronomical Society. For the full-color figure, see that paper.

the corresponding polarization was due to asphericity of the SN ejecta. By contrast, the polarization at the $2012 \mathrm{~b}$ peak must arise from a different scattering region. Attributing this second 2012 brightness peak to circumstellar interaction, we proposed the circumstellar material was equatorially concentrated orthogonal to the ejecta axis (Figure 2). This geometry is similar to the one suggested by Levesque et al. (2014) based on spectral modeling. This CSM likely arose from the progenitor's mass loss in late evolution, and its flattened nature could indicate a binary origin.

\section{The Future of Supernova Spectropolarimetry}

The technique of spectropolarimetry applied to SNe is poised to enter a new era characterized by more and more frequent observations as well as increased wavelength coverage. This wealth of data will drive continued advances in spectropolarimetric modeling and give rise to a new dimension in our understanding of SNe. In particular, the combination of larger statistical samples with more detailed modeling will reveal new information about the structure and evolution of different types of SNe, as well as illuminating currently obscure connections among different SN subtypes. In turn, these developments will catalyze a fuller understanding of the late stages of stellar evolution and the relationships among progenitors and different SN classes.

The status of spectropolarimetric instrumentation on the next generation of large telescopes is somewhat uncertain. Polarimetric capabilities are being discussed in the context of the Giant Magellan Telescope (GMT) and Thirty Meter Telescope (TMT), but need strong community support to have a chance of succeeding. In the meantime, 

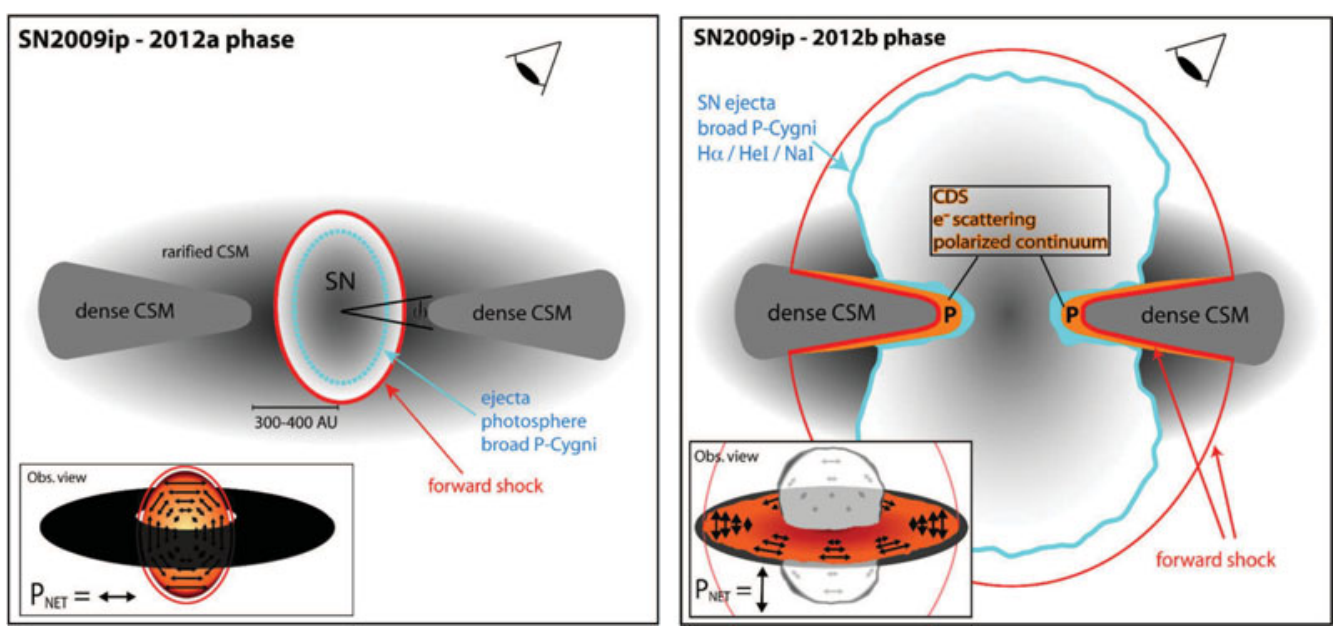

Figure 2. Left: Illustration of a potential SN/CSM configuration for SN 2009ip during the 2012a event (not to scale), before the onset of intense CSM interaction. The detection of significant continuum polarization at this phase suggests an aspherical geometry for the SN photosphere and/or partial absorption by toroidal CSM (inset). The approximate orthogonality of the polarization position angle with respect to the $2012 \mathrm{~b}$ CSM interaction phase suggests that the SN photosphere might have a bipolar geometry. Right: Illustration of a possible configuration for the $2012 \mathrm{~b}$ phase, after the onset of intense CSM interaction. A toroidal distribution of dense CSM gives rise to strong shocks, electron scattering, and a luminous polarized continuum (marked by a bold-faced 'P'). Figure from Mauerhan et al. (2014), courtesy Oxford University Press and the Royal Astronomical Society. For the full-color figure, see that paper.

however, the Robert Stobie Spectrograph (RSS), built by the University of Wisconsin for the 11-m Southern African Large Telescope (SALT) is restarting spectropolarimetric commissioning after instrument repairs in 2014; SALT's large collecting area combined with RSS's high resolution and UV sensitivity will contribute significantly to both stellar and SN polarimetry in the near future (Nordsieck 2012).

\section{Acknowledgements}

Many thanks to the International Astronomical Union, the High Altitude Observatory, and the University of Denver's Office of Internationalization for supporting my participation in this symposium. The SNSPOL project is supported by NSF awards AST-1210599 (U. Arizona), AST-1210311 (SDSU), and AST-1210372 (U. Denver); the SN 2009ip research was also partly funded by NSF award AST-1211916 (UC Berkeley).

\section{References}

Chornock, R., Filippenko, A. V., Li, W., \& Silverman, J. M. 2010, ApJ 713, 1363

Davidson Jr., J. W., Bjorkman, K. S. Hoffman, J. L., et al. 2014, JAI 03, 1450009

Dessart, L. \& Hillier, D. J. 2011, MNRAS 415, 3497

Filippenko, A. V. 1997, ARAA 35, 309

Foglizzo, T., Kazeroni, R, Guilet, J., et al. 2015, PASA, in press (astro-ph:1501.0134)

Fraser, M., Magee, M., Kotak, R., et al. 2013, ApJ 779, 8

Hoffman, J. L., Leonard, D. C., Chornock, R., Filippenko, A. V., Barth, A. J., \& Matheson, T. 2008, ApJ 688, 1186

Hoffman, J. L., Smith, N., Bilinski, C., et al. 2014, AAS Meeting Abstracts 223, 354.21

Huk, L. N., Peters, C. L., \& Hoffman, J. L. 2013, AAS Meeting Abstracts 221, 253.16

Kasen, D., Nugent, P., Wang, L., et al. 2003, ApJ 593, 788 
Kawabata, K. S., Jeffery, D. J., Iye, M., et al. 2002, ApJ 580, L39

Khandrika, H. G., Leonard, D. C., Horst, C., et al. 2014, AAS Meeting Abstracts 224, 121.16

Leonard, D. C., Filippenko, A. V., Chornock, R., \& Li, W. 2002a, AJ 124, 2506

Leonard, D. C., Filippenko, A. V., Li, W., et al. 2002b, PASP 114, 35

Leonard, D. C., Filippenko, A. V., Ganeshalingam, M., et al. 2006, Nature 440, 505

Leonard, D. C. 2011, Ap\&SS 336, 117

Leonard, D. C., Dessart, L., Hillier, D. J., \& Pignata, G. 2012, in: J. L. Hoffman, J. Bjorkman, \& B. Whitney (eds.), Stellar Polarimetry: From Birth to Death, AIP Conf. Ser. No. 1429 (New York: AIP), p. 204

Levesque, E. M., Stringfellow, G. S., Ginsburg, A. G., Bally, J., \& Keeney, B. A. 2014, AJ 147, 23

Margutti, R., Milisavljevic, D., Soderberg, A. M., et al. 2014, ApJ 780, 21

Mauerhan, J. C., Smith, N., Filippenko, A. V., et al. 2013, MNRAS 430, 1801

Mauerhan, J., Williams ,G. G., Smith, N., et al. 2014, MNRAS 442, 1166

Maund, J. R., Wheeler, J. C., Patat, F., Baade, D., Wang, L., \& Höflich, P. 2007, MNRAS 381, 201

Milne, P., Williams, G. G., Smith, P. S., \& Smith, N. 2014, AAS Meeting Abstracts 223, 354.39

Nordsieck, K. H. 2012, in: J. L. Hoffman, J. Bjorkman, \& B. Whitney (eds.), Stellar Polarimetry: From Birth to Death, AIP Conf. Ser. No. 1429 (New York: AIP), p. 248

Patat, F. Taubenberger, S., Cox, N. L. J., et al. 2015, A\&SA, submitted (astro-ph:1407.0136)

Schmidt, G. D., Elston, R., \& Lupie, O. L. 1992, AJ 104, 1563

Tsebrenko, D. \& Soker, N. 2015, MNRAS 447, 2568

Turatto, M., Benetti, S., \& Pastorello, A. 2007, in: S. Immler, K. W. Weiler, \& R. McCray (eds.), Supernova 1987A: 20 Years After: Supernovae and Gamma-Ray Bursters, AIP Conf. Ser. No. 937 (New York: AIP), p. 187

Van Dyk, S. D. 2011, in: P. Roming, N. Kawai, \& E. Pian (eds.), Death of Massive Stars: Supernovae and Gamma-Ray Bursts, IAU Symp. No. 279 (Cambridge: Cambridge University Press), 7, p. 110

Wang, L. \& Wheeler, J. C. 2008, ARAA 46, 433

Williams, G., Dessart, L., Hoffman, J. L., Huk, L. N., Leonard, D. C., Milne, P., Smith, N., \& Smith, P. S. 2014, AAS Meeting Abstracts 223, 354.22 